\section{CONTRIBUIÇÃO AO ESTUDO DAS VIAS BILIFERAS INTRA E EXTRA-HEPÁTICAS EM CAVALOS, PURO SANGUE INGLÊS. SISTEMATIZACĀO DO DUCTUS CHOLEDOCUS E RAMUS PRINCIPALIS DEXTER}

ANGELO JOAO STOPIGLIA Professor Assistente Br. Faculdade de Medicina Veterinaria e Zootecria da USP

ANTONIO FERNANDES FILHO Professor Titular Faculdade de Medicina Veterinaria Zooteenia da USP

NILSON FERREIRA Professar Adjunto Faculdade de Medicina Veterinaria e Zootecnia da USP

STOPIGLIA, A.J.i FERNANDES FILHO, a.; FERREIRA, M. Contribuicto ao estudo das vias billferas intra: extra-nepaticas en cavalos Puro Sangue Ingles. Sistematizacso do duetus chaledocus. ranus principalis dexter. Rev. Fac. Med. vet.

zootec. Univ. 5. Paulo, 23 (2):103-113, 1986.

RESUMD: Estudando sistematizacso do duetus choledocus pamus princioalis dexter, en 30 flgados de eavalos P.S.I. 20 machas e 10 remeas, adultas, ehegou-se is seguintes canctuszes: (1) ductus choledocus encontri-se na majoria das pecas ( 36,75$)$, formada pe!a uniza do panus prinelpalls dexter pamus principalis sinister, enquanto nas restantes preparactes. pesulta de triplice convergancia do ramus prineipalis dexter, pans princlpalis sinister pamus lodi quadrati (30.05) ou, do ranus prinejpalis dexter. ramus princlpalis sinister. pamus ventralis bobi dextrl (6,75); 2) o sistema do ramus princidolis dexter aparece constituldo pelo ramus dorsalis lobl dextri panus nedius looi dextri, pamus rentrabls lobl dextri, pamus processi caudati. pamus lobi quadrati aferentes inominados do labus dexter (terpitoplos dorsal, medio ven(rab), do (abus saudatus (processus eaudatus). do lobus quadratus.

UNITERMOS: Anatonia, equinosi Flgadei Ductos bltiares

\section{INTRODUCAO E LITERATURA}

0 estudo do sistema biblfero mostrase, hodierriamente, como enorme camoo de pesquisa, em especia! quando buscamos conhecimentos mais detalhados, no que respejta is mais imoortantes vias de drenagem do flgado em animais desprovidos de vesi. cula blliar, mais precisamente, o savalo Puro Sangue Ingles.

No tocante aos livros de texto consultados esclarecemos que as informactes apresentam-se genericas e imprecisas, restringindo-se, quase semore, a constituicso do ductus eholedocus.

Assin, MONTANE \& BOURDELLE (16), LEPOU-

TRE (13), LESBRE (14), a i rmam que as vias billferas encaminham-se para a porta hepatis, onde formam o canal coledoco, comportamento tamben verificado por BOURDELLE \& BRESSDL (i), relativamente a eqdideos. FAUILLI (7), e GONZALEZ y GARCIA \& GONZALEZ RLUAREZ (10) discordam dos autares precedentes, apenas no concernente a nomenctatu$r a$ is to e, denominam-no, respectivamente, oucto hepato-entérico e conduto coledoco. SCHUMMER \& NICKEL (18) , KOCH (12) SCHWRRZE \& SCHRODER (19) descrevem aue os ductus billferi se rednem para dar origem ao ductus hepaticus; este torna-se calibroso no trato final e, sem qualquer delimitacro, é chanado de ductus choledocus. ZANOLLI (21) a ELLENBERGER \& BAUM (B) assinalam a presenca de dois troncos confluentes, direito e esquerdo, sendo este altimo mals calibroso, visto a receber coletor dos lobos esquerdo medio. Uma vez configurado a ductus hepatlcus, estes autores designan o segmento final do aludido ducto, de ductus choledocus. BRRDLEY (2) considera sinonimos os termos dueto billfero coledoco, relatando, ainda, que - ducto hepatico mais consplcuo drena os lobos lateral central esquerdas, enquanto - outro ducto acolhe os coletores do lobo lateral direito. BRUNI \& ZIMMERL (3), ao se referirem is vias billferas, falam em conduto hepatico e que a via escoadora do lobo medio abre-se na raiz esquerda deste. Para SISSON \& GROSSMAN (20) o conduto hepatico resulta da unizo dos principais condutos lobares direito e esquerdo. enquanto, de sua parte, GETTY (9) menciona que o ducto hepatico comum e formado, na porcso ventral da incisura hepatiea, pela unizo dos ductos hepáticos direito esquerda. Por fim, MARTIN (15) declara que o dueto soledoco tem origem triplica, d lsposicgo tambem relatada por CARADONNA (4) que, entretanto, denomina o aludido ducto de canal ou canduto coledoco.

Relativamente aos trabalthos especiali. $z$ ados, verificamos que OTTRUIANI (17) ao estudar as vias biliferas intra-hepaticas, em cavalos (adultas e fetos), sem citar o numero de animais utilizados, mediante andlise radiografica, informa que o plano sagital situado em correspondencia ao eixo 
do conduto coledoco, divide nitidamente o orgyo en duas seceres, uma direita abrangendo os lobos direito e caudado, outra esquerda, compreendendo os lobos esoueroo, mediano (tobo quadrado) e a Dar te escuera do caudado.

O ducto billfero direito, acrescenta o autor, resulta da unigo em vu de dois af luentes primarios, provenientes dos lobos direito e caudado. Este ducto recebe dois ou tres afluentes primarios ventrais e lgual numero de dorsais, fazendo parte deste ultimo grupo os do lobo caudado. Ainda, um longo afluente primbrlo, como ben mostra a figura que ilustra o trabalno, nasce da zona compreendida entre os territorlos dos dois afluentes que associados forman $D \cdot v$, atingindo o ducto billfero direito, pela sua poreso dorsal. Un outro, procedente das regibes mediana e ventral do lobo direito, dirige-se a porta hepatis e abre-se no coledoco. Dos dois ou tres a fluentes vindos do lobo caudado, um concorre para formar a dueto bilifero direito e um ou dois lancam-se no contorno dorsal do seu trato terminab.

D lobo mediano, refere-se o autor, mostra dois ou tres afluentes primbrios, sendo que dois deles desembocan no dueto billfero esquerdo o o terceiro gente o segmento inicial do colddoco.

R seu turno, JRBLRN-PANTIC (11) estudando comparativamente as vias biliferas intra-nepaticas de 58 animais domésticos, examina $B$ flgados de equinos, de ambos os sexose de diferentes racas e idades, apos injecro das pecas, atraves do ductus choledocus, con substancia radiapaca, sequida de $f i x a c 50$ pela formalina 4,08, oferecendo as seguintes conclustes:

a) o ductus hepatlcus, cuja secero distal representa o ductus choledocus, fornece dois ramos, ou seja, o ramus principalis dexter e o ranus principalis sinister:

b) o ramus principalis dexter drena o lobus dexter mais o processus caudatus, por intermedio de un ramo ventral e de tres a cinco ramos laterais. dorsais - rami lobl dextri, servindo as porsoles fiomonimas daquele lobo, apds o que penetra no lobus caudatus, cono ranus processi caudati.

DUPRAT (5), trabalhando com $105 \mathrm{fl}$ gados de eqúlinos, sendo 50 deles destinados ao exame das vias biliferas, com auxilio da disseccso e corrosto, apresenta os resultados que em parte transcrevenos:

1) o canal coledoco resulta da unigo do ductus hepatieus dexter e do duetus hepaticus sinister, responsaveis pela drenagem dos correspondentes territorios direito e esquerda;
2) o sistema dipelto drena o Lobo dareito (segmento $V$ e VI) e o processo caudado (segmento VII):

3) o lobo direito (seamentos VeVIr e drenado pelas palzes de cada um dos dois segmentos, ou seja, por uma raiz dorsal e outra lateral do segmento VI e por duas ratzes ventrais do segmento $V_{i}$

4) via de escoamento do processo caudado (segmento VII) une-se a sistema direito en 43 observacses, desemboca diretamente no canal coledoco 6 vezes, mostrando-se ausente na peca restante.

FERREIRA ( 8 ), oo pesouisar as vias billferas intra e extra-hepaticas no Equus caballus, utilizou 30 animais de raca nao definida (SRD), de ambos os sexos e de diferentes e ngo conhecidas idades. Rpos injeczo atraves do ducto coledoco, com "Neodrene latex E50" adicionado de Celobar - 50,0\% e fixacyo em soluego de formol a $10,0 \%$, as pecas for an dissecadas, esquematizadas e fotografadas, chegando a autor as seguintes conclusoles:

a) o ductus choledocus que, en 23 pecas $(76,7 \%)$; resulta da fusto do panus principalis dexter e ranus principalis sinister, visto tambam, por ordem de freqoencia, a originar-se de trlplice convergencia: do ramus princlpalis dexter, ramus principalis sinister e ranus ventralis lobi dextri, 3 vezes $(10,08)$, do ranus principalis dexter, ranus principalis sinister e ranus lobi quadrati, 2 vezes $(6,78)$; do ramus principalis dexter, ranus principalis sinister e ramus dorsalis lobi dextri, 2 vezes $(E, 7 \%)$. Do conjunto de disseccres no qual o focado ducto e constituldo pelo ramus principalls dexter e ramus principalis sinister, observou a receber, $q$ vez $(3,38)$, o pamus ventralis lobi dextrl;

b) Livre de afluentes, na quase totalidade dos casos, ou seja, em 29 deles $(96,78)$, o ductus choledocus acolhe, 1 vez $(3,3 \%)$, mediante tronco comum, duas vias de drenagem procedentes dos territorios medio e ventral do lobus dexter:

c) o sistema do ramus principalis dexter d formado pelo ramus ventralis lobi dextrl, ramus medius lobl dextri, ramus dorsalis lobi dextri, pamus procassi caudati e coletores inominados do lobus dextar lterpitorios medio, dorsal e ventral), do lobus caudatus (processus caudatus), do lobus quadratus e do lobus sinister lateralis (territario dorsal); 
- ramus principalis dexter ten por ralzes, en conbinacbes sucessivas as seguintes vias: 19 vezes $(36,78), 0$ ramus medius lobl dextef, o panus processi caudati, o ramus dorsalis Lobi dextri e o ramus ventralis looi dextrl; 4 vezes $(13,38)$, o panus medius lobi dextri, o pamus dorsalis lobi dextri, o panus processi caudati a o ramus ventralis lobi dextri; vezes $(13,3 \%), 0$ ramus medius lobl dextri, a ramus processi caudati e o camus dorsalis lobi dextri; 2 vezes $(6,78)$, o pamus medius lobl dextrl, o camus processi caudati e o pamus ventralis lobi dextri; 2 vezes $(6,78)$, o ramus medius lobi dextri e o ramus processl caudatl a que se associan, por tronco, o ranus dorsalis lobl dextri o ramus ventralis lobi dextpi; 2 vezes $(6,78)$, o pamus dorsalis lobi dextri a o ramus medlus lobi dextri, unidos a tributários para o qual concorrem o ramus ventralis lobi dextri a o ramus processi caudati; $?$ vez $(3,3 \%)$, o pamus dorsalis lobi dextri, o ramus processi caudati e - ramus medius lobi dextri; 1 vez $(3,38)$, o ramus dersalis lobi dextri, - ramus processí caudati, a ramus medius lobi dextrleo pamus ventralis lobl dextrl; 1 vez $(3,38)$, a paus medius lobi dextri, o ramus processi caudat 1 , o ramus dorsalis lobi dextri. - pamus ventralis lobl dextri o pamus lobl quadrati; 1 vez $(3,3 \%)$, 0 pamus medius labi dextrle o pamus processi caudati. Ein 1 dos drgtos (3,38), ha tpiplice fuszo do panus dorselis lobi dextri, ramus processi caudati e ramus nedius lobl dextri. em cuja via desemboca o ramus ventralls lobi dextif;

e) uma vez individualizado, o panus principalis dexter, livre de aferentes 11 vezes $(36,78)$, recebe nas outras 19 $(63,38)$, contingentes vindos: apenas do lobus dexter, 6 vezes; concomitantemente do lobus quadratus do lobus caudatus, 6 vezes; simultaneamente do lobus dexter (territorio ventral) e lobus quadratus, 3 vezes: conjuntamente do lobus dexter (territorio ventral) lobus saudatus, 2 vezes; a un so tempo do lobus dexter (territorio ventral), lobus quadratus e lobus caudatus, 1 vez; concomitantemente do lobus dexter (territorio ventrab), lobus quadratus, lobus caudatus, labus sinister lateralis cterritorio dorsal), i vez;

f) na constitulfao do ramus principalis dexter ductus choledocus, nja foram registradas diferencas estatisticamente significantes, quando confrontados os sexos.
MATERIAL E METODO

Para a presente pesquisa valemo-nos de 30 pecas, comoreendendo, cada uma, flgado e segmento ouodenal, retiradas em bloco, de eqóinos Puro 5 angue Ingless, sendo 20 machos $(m)$ e 10 femeas ( $f$ ), adultos, de diferentes idades, procedentes do Jockey Club de 590 Paulo da Faculdade de Medicina Veterinaria Zooteenia da U.S.P.

Una vez isolado o conjunto, aberto o duodeno ao longo de sua borda livre e exposta a mucosa, canalizamos o ducto coledoco atraves da papila duodenal major e esvaziamos, tanto quanto possivel, o sistema billfero a custa de massagens; injetamolo, entro, com Neoprene latex* $450^{\circ}$ adicionado de Celobar** na proporeyo de 50,0\%. Depois de radiografadas e fixadas em solucga aquosa de formol a 10,08 , as glandulas foram sucessivamente dissecadas, fotografadas e esquematizadas para ulterior andise.

Os territorios hepaticos, nesta especie. mostram-se parcialmente delimitaoos por incisurase, para facilitar o relato dos resultados, convencionamos, como sugeren SCHUMMER \& NICKEL (18) demarcar a flgado por meio de dois planos imaginarios, perpendiculares ds superfleies diafragmbtica - viscaral do orgro. Um, imaginamo-lo acompanhando o maior eixo da fissura hepbtica direita ate atingir, dorsalmente, o sulco da veía cava caudali o outro, idealizamo-lo a percorrer a incisura correspondente ao ligamento redondo ate eruzar - lapressgo esofbgica. Deste forma, a direita do primeiro plano encontram-se o lobus dexter, uma pequena parte do lobus caudetus e o processus caudatus a a esquerda do segundo, situan-se o lobus sinister lateralis e o lobus einister medialis. Mo segmento intermediario apresentam-se, ventralmente, o lobus quadratus e, dorsalmente, porcyo do lobus eaudatus correspondente a pars supraportalis.

No curso das disseceras foi-nos possivel, ainda, estabelecer divisoles das regibes direita e esquerda do figado; definimos, assim, na superfleie visceral do orgoo, tres territorios dispostos radlatmente, ou seja, dorsal $(1 / 4)$, medio $(2 / 4)$ e ventrat $(9 / 4)$.

Na sistenatizaço das vias billferas, Levamos en conta a extensăo dos territorios escoa'dos mais que o calibre dos ductos, pelo fato das injectes preencherem melhor os de situacro superficial. Cabe, ainda, eselarecer que deixamos de registrar os pequenos tributarios dos ramos conspleuos, somente quando drenavam as mesmas regiaes hepaticas dos aludidas pamos, nazo 5 as sociando, contribuicao de procedencia diversa.

Para efeito de relato, obedecemos o sentido do fluxo secretorio da bile, ou seja, periferia-centro; respeitamos a iden- 
tidade dos ramos nominados ate sua associacro con outro de igual categoria ou com tronco, por vezes inominado, resultante da sua confluencia.

Na aoresentacóo dos resultados, emoregamos a nomenciatura utibizada dor JABLANPANTIE (11) com uma ressalva, vale dizer, relacionada com o eriterio adotado na designacgo do duetus choledocus.

Finalmente, esclarecemos que aplicamos o teste do $x^{2}$ no estudo estatistico dos nossos dados.

\section{RESULTADOS}

Nossos resultados, concernentes à sistematizacgo das vias billferas no cavalo Puro Sangue Ingles, dizen respeito, dois ltens, is to :

R - Ductus choledocus

1. o auctus sholedocus aue em 19 casos (53,3x - Fig. 3) e visto formado pela unigo do ramus principalis dexter e pamus principalis sinister, resulta, nos 11 outros $(36,78)$, do triplice convergencia do pamus prineipalis dexter, ramus principalis sinistere ramus lobi quadrati, $g$ vezes $(30,08$ Fig. i) ou do ramus principalis dexter, ramus principalis sinister e ramus ventralis lobi dextri, 2 vezes $(6,78$ - Fig. 2$)$;

2. Identificado livre de tributbrios en 23 preparactes $(76,7 \%)$, o ductus choledocus recebe nas mais $7(23,3 \%)$, contribuicoes: apenas do lobus quadratus, 3 vezes $(10,0 x)$; do lobus quadratus a labus dexter (territorio ventral) associados, 2 vezes $(6,7 \%)$; unicamente do lobus dexter (territorio ventral), 1 vez $(3,3 \%)$ a ainda, ramus ventralis lobi dextri, $q$ vez $(3,38)$;

\section{B - Ramus principalis dexter}

9. o sistema do ramus principalis dexter é constituldo pelo ramus dorsalis labi dextri, ramus medius labi dextri. ramus ventralis lobi dextri, ramus processi caudat1, ramus lobi quadrat e aferentes inominados provenientes do labus dexter (territarios dorsal, médio e ventral), do lobus caudatus (processus caudatus) e do lobus quadratus:

2. o ramus principalis dexter tem por ralzes, en combinacós sucessivas, as seguintes vias: 18 vezes (60,0\%Figs. 1,4$)$, o ramus medius lobi dextri, o ramus processi caudati, o pamus dorsalis lobi dextri e o ramus ventra- lis lobi dextri; 3 vezes $(10,0 \%$. Figs. 3), o ramus medius labi dextri, - Pamus processi caudati e o ramus ventralis lobi dextri; 2 vezes ( $(5,7 \%)$, o ramus medius lobi dextri, o ramus processi caudati, o ramus ventrabls lobi dextri e o ramus dorsalis lobi dextri; 2 vezes $(6,78$ Fig. 2) o ramus medius lobi dextri, o ramus processi caudati e o ramus dorsalis lobi dextri; 1 vez $(3,3 \%)$, o ramus medius lobi dextri, o ramus dorsalis lobi dextrl, o panus processi caudati e o ramus ventralis lobi dextri; 1 vez $(3,3 \%)$, o ramus medius labi dextri, o ramus dorsalis labi dextri e o ramus processi caudati; 1 vez (3,38), o pamus medius lobi dextri, o ramus ventralis lobl dextri, - pamus processi caudati e o ramus dorsalls lobl dextrli 1 vez $(3,35), 0$ ramus dorsalls lobi dextrl, o ramus processi caudati, o ramus medius lob: dextrl e o ramus ventralis lobi dextri; 1 vez $(3,3 \%), 0$ pamus medius lobi dextri, o ramus processi caudati, - ramus dorsalis lobi dextri, o ramus ventralis lobi dextri e o ramus lobi quadrati;

3. ume vez individuolizado, o pamus principalis dexter, livre de tributarios 91 vezes $(36,7 \%)$, perebe nas outras $19(63,3 \%)$, contingentes oriundos: apenas do lobus dexter (terribrio ventral), 9 vezes; somente do lobus caudatus, 6 vezes; exclusivamente do labus quadratus, 1 vez simultaneamente do lobus dexter (territorio ventral) e lobus quadratus, 1 vez; concomitantemente do lobus dexter (territorio ventral) e lobus caudatus, 1 vaz e conjuntamente do lobus cauastus e lobus quadratus, 1 vez;

4. na constituicyo do ductus choledocus e do ramus principalis dexter nao foram registradas diferencas estatisticamente signifleantes, a nivel de 5,0\%, quando confrontados os sexos.

\section{COMENTARIOS E CONCLUSOES}

Ds autores dos varios tratados de Anatomia Veterinaria reportam-se unicamente a principal via de escoamento do flgado de eqdinos, e nomeiam-na de ductus choledocus (2, 15), conduto hepatico $(3,20)$, dueto bilifero (2), canal colédoco $(1,4,13,14,16)$, conduto coledoco $(4,10)$, ducto hepato-enterico (7) e ducto hepatico comum ( 9 ). 0 duetus eholedocus $d$ assim denominado por alguns $(E, 12,18,19,21)$, ape- 
nas em correspondencia ao segmento distal do ductus hepaticus.

No atinente ds vias responstueis pela formaczo do ductus choledocus, encontramo(as deseritas como troncos ( $5,2 i)$, duetos hepaticas $(2,9)$, paizes ( 3 ), condutos lobares $(10)$, e canais oiliares principais (4). Cabe destacar que somente certos AR. $(4,15)$ fazem mencso j opigen tplplice do ductus choledocus.

No que tange aos trabalhos especializados devemos, inicialmente, fazer ressalva quanto a nomenclatura empregada por JABLANPANTIC ( 19 ), apenas no concernente ao criterio adotado na designaca do ductus choledocus, porque em outras especies, en que a vesicula biliar esty presente, o ductus cysticus ora se integra ao ramus principalis dexter ora ao ramus principalis sinister.

Para OTTRUIANI (17), o plano sagital correspondente ao eixo do conduto coledoco, divide o flgado nas territorios billferos direito (lobo direito, lobo caudado) e esquerdo (lobo esquerdo, lobo mediano ou quadrado e a parte esquerda do lobo caudado), drenados, respectivamente, pelos duetos biliferos direito esquerdo, isto e, ramus prineipalis dexter a pmus principalls sinister.

DUPRAT (5), estudando a sistematizac8o dos canais biliares, informa que o canal coledoco e constituldo pelos ductus hepaticus dexter (ramus principalis dexter) e ductus hepaticus sinister (ranus principalis sinister), responstreis pelo escoanento da bilis dos territorios direito e esquerdo.

Jd, FERREIRA ( 8 ) af irma que o ductus choledocus resulta da unigo do ramus princlpalis dexter e do ramus principalis sinister, em 76,78 dos casos, fato comprovado em 63,3\% de nossas pefas; outras modalidades 5 go verificadas pelo citado autor, vale dizer, a da constitulgao por triplice convergencia, envolvendo ora as mencionadas vias e pamus ventralis lobi dextri $(10,08)$, os dois primeiros ductos a ramus lobi quadrati $(6,75)$, ou, o ramus principalis dexter, o ramus principalis sinlster e - ramus dorsatis lobi dextri $(5,78)$, disposic8es por nos registradas, respeitada a sequencia, em 6,78 e $30,0 \%$ das preparacbes. Cabe destacar que ngo assinalamos a ultima modalidade.

Una rez configurado o ductus choledocus, atTauianI (17) abserva que un contingente primario do territorio esquerdo (lobo medianol e outro do territorio direito (porcoles mediana e ventral do lobo direita) desembocam no aludido ducto, enquanto DUPRAT (5) informa = =hegada de via pesoonshuel pela drenagem do processo caudado do ouctus choledocus em $12,0 \%$ dos flgados. FERREIRA ( 8 ) tambem observa que o referido ducto recebe, em $3,3 \%$ dos casos, contribuibes procedentes do lobus dexter (territorios medio e ventral) e ramus ventralis labi dextri. Em 23,38 de nossas pecas identificamos o ductus choledocus acolinendo contribuicóes do lobus quadratus, do lobus dexter (territorio ventral) e o ramus ventralis lobi dextr!.

Entrando, agora, ao apreziar o proolema da sistematizaço do ramus principalis dexter, vimo-lo, de modo geral, a drenar o lobus dexter, o lobus caudatus e o lobus quadratus, por intermedio do ramus dorsalis lobi dextri, ramus medius lobi dextri, ramus ventralis lobi dextri, ramus processi caudati e ramus lobi quadrati, afora contribuictes inominadas oriundas de todos os territorios mencionados. Para FERREIRA ( 8 ). - ramus principalis dexter, além de acother a bile dos territorios acima relacionados, excetuando-se o lobus quadratus (ramus lobi quadrati), recebe tributario inominado do lobus sinister lateralis iterritorio dorsal). Por sua vez, JABLAN-PANTIC (11) relata que, o pamus prinelpalls dexter drena a bile do processus caudatus e lobus dexter, gracas ao ramus processi cauati. pamus ventralis looi dextri e, de tres a cinco rami lobi dextri (partes laterais e darsais do labus dexter) eorrespondentes estas altimas vias as que designamos por ranus medius lobi dextri, ramus dorsalis lobl dextri, contingentes inominados vindos do lobus dexter (territorios dorsal e medio) e do lobus caudatus. D pamus processi caudati configura, segundo af i rmam JABLANPANTIC (11) e FERREIRA (8), com o que concordamos, a propria continuacgo do ramus principalis dexter. Para DUPRAT (2), o ductus hepaticus dexter (ramus principalis dexter) escoa a bile do lobo direito (segmentos $V$ a VI) e processo caudado (segmento VII), vale dizer, lobus dextar e processus caudatus resoectivamente. A drenagen do lobus dexter é realizada, segundo andlise do esquema apresentado pelo autor, por uma raiz dorsal, outra lateral e por mais duas palzes ventrais, designadas segundo a nomenclatura que utibizamos, obedecendo a orden, ramus dorsalis lobi dextri, ramus medius lobi dextri, ramus ventralis lobl dextrl, enquanto o escoamento do processo caudado efetuado por via correspondente ao ramus processi caudati. obedecendo o mesmo criterio.

De outra parte, OTTRUIANI (17) infarma que o ducto billfero direito (ramus principalls dexter) nasce da unizo em ' $V$. de dois afluentes primarios provenientes do lobo direito (ramus medius lobi dextri e contribuicoles inominadas do territorio dorsal) e do lobo caudado (eoletores inominados do (obus caudatus). Verificando-se a documentacao exibida por este autor, notamos que a via assim formada vem ter o ramus arocessi caudati, para depois chegar a ramus dorsalis lobi dextri, comportamento observado Dor FERREIRA em 13,3\% dos casos a ngo encontrado em nosso material.

Da mesma forma, examinando figura inclulda na publicacáo de JABLAN-PANTIC (11), ja que este nao descreve as as- 
sociacbes entre os ductos, surpreendemos o ramus processi caudati associado a ramus medius lobj dextri, configurando tronco para o qual convergem, por ordem, o ramus dorsalis lobi dextri e o pamus ventralis looj dextri. Esta mooalioade e observada em $60,0 \%$ das nossas pecas em 36,78 das de FERREIRA ( 8 ).

Analisando, igualmente, o esquema de distribuifgo padryo das vias billferas inserido por DUPRAT (5) em sua tese, verificamos o ramus processi caudati aliado ao ramus dorsalis lobi dextri, constituindo tronco do qual chegan, sucessivamente, o ramus medius lobi dextrl e o ramus ventralis lobi dextri, comportamento visto por nos e por FERREIRA ( 8 ) com a mesma frequencia, isto e, en $3,3 y$ dos flgados dissecados.

Cotejando nossos actrados com os de FERREIRA ( $B$ ), no concernente is diferentes modalidades de combinacbes dos ductos conspleuos na organizacalo do ramus principalis dexter, enumeraremos as assocjactes e respectivas frequencias assinaladas por nose nyo registradas pelo aludido autor, vale dizer; o pamus medius lobi dextri, ramus processi caudati, pamus ventralis lobi dextri a ramus dorsalis lobl dextri ( $5,7 \%)$, o panus medius lobi dextr1, panus dorsalis lobi dextri o panus processi caudati (3,38); o panus medius. Labi dextri, ramus ventralis lobi dextri, ramus processi caudati e o ramis dorsalis lobi dextri $(3,34)$. For outro lado, cabe relatar, da mesma forma, os arranjos identif:cados com suas percentagens na tese de FERREIRA ( 8 ) e ausentes en nossa investigasyo, isto $b$, o panus nedius lobi dextri o ramus processi caudati, formando via qual se associam, por tranco, o ramus dorsalis lobi dextri e o ramus ventralls lobl dextri $(E, 7 \%) ; 0$ ramus dorsalis lobi dextri, pamus medius looi dextri, ramus ventralis lobi dextri e o panus processi caudati $(6,7 \%)$; - ramus dorsalis lobi dextri, pamus processi caudati o ramus medius lobi dextri (3,38); o panus medius lobi dextri e o ramus processi caudati $(3,3 \%)$; a triplice fusgo do ramus dorsalis lobi dextri, ramus processi caudati, pamus medius lobi dextri e o ramus ventralls lobi dextri $(3,35)$.

Ainda, OTTAUIANI (17) diz que o dueto billfero direito (ramus principalis dexter) una vez constituldo, recebe dois ou tres afluentes primarios de origem ventral e igual numero de procedencia dorsal, os quais correspondem en nosso trabalho, aos ranl medil lobl dextri e, ao panus processi caudati, ramus dorsalis labi dextri, afora contribuiclos inominadas- do lobus dexter (terpitorio dorsal) e do lobus caudatus.
Quanto so mesmo aspecto, FERREIRA ( 8 ) encontra o ramus principalis dexter, depois de formado, livre de afluentes em 36,75 dos casose, nos 63,3\% a acolher contribuifares do lobus dexter (territorio ventral), lobus caudatus, lobus quadratus e lobus sinister lateralis (territorio dorsal), confirmados por nós, com a ressauva de ná assinabarmos a participacgo de contribuiczes do lobus sinister lateralis.

Finalmente, analisando os resultados ora obtidos en cavalos da raca Puro Sangue Ingles, com aqueles assinalados no SRD, mediante o emprego do $x^{2}$, verificamos diferencas estatisticamente significantes a nivel de 5,0\%, nos seguintes casos: a) formacto do ductus choledocus pela triplice convergencta do ramus princlpatis dexter, ramus principalis sinister e ramus lobi quadrati, b) frequancia de contribuictes recebidas pelo ductus choledocus, apos individualizado; c) conflguraço do pamus principalis dexter, quando a eitada via era formada, por ordem, pelo ramus medius lobi dextri, ramus processi caudati, ramus dorsalis lobi dextri e ramus ventralis booj dextri.

STOPIGLIA, R.J.: FERMANDES FILHD, A.; FERREIRA, N. Contrloulion to the study of the biliory systen in Throughbred horses. Systematization of the ductus choledocus and the ramus principalis dexter. iev.Fac. Med.vet. Zootac. Univ.5.Paulo, 23 (2):103-113, 1986.

SUMmaRY: In a study about the systematization of the ductus enoledocus and the panus principalis dexter, in 30 livers of adult, 20 male and 10 females, there vere arpitved to the following canclusions: i) the duetus choledocus is formed by the ranus pplincipalis dexter and ramus prineipalls sinister $(36.7 \%)$, by the ramus principalis dexter, ramus principalis sinister and pamus lobi quadpati (30.0s), by the pamus peincloalls dexter, pamus princioalis sinister and ramus ventpalis loob dextri (6.78); 2 ) the systen of the panus principalis dexter is comoosed by the - anus darsalis lobl dextrl. pamus aedius lobi dextri. paaus ventpalis lobi dextri, ramus processi caudat1, ramus lobi quadpati and Inominated afferents of the lobus dexter coorsal, aedial and ventpal terpitopies), lobus caudatus (processus caudatus and lobus quadratus.

UWITERMS: Anatony of equines: Wiver; Bile ducts 


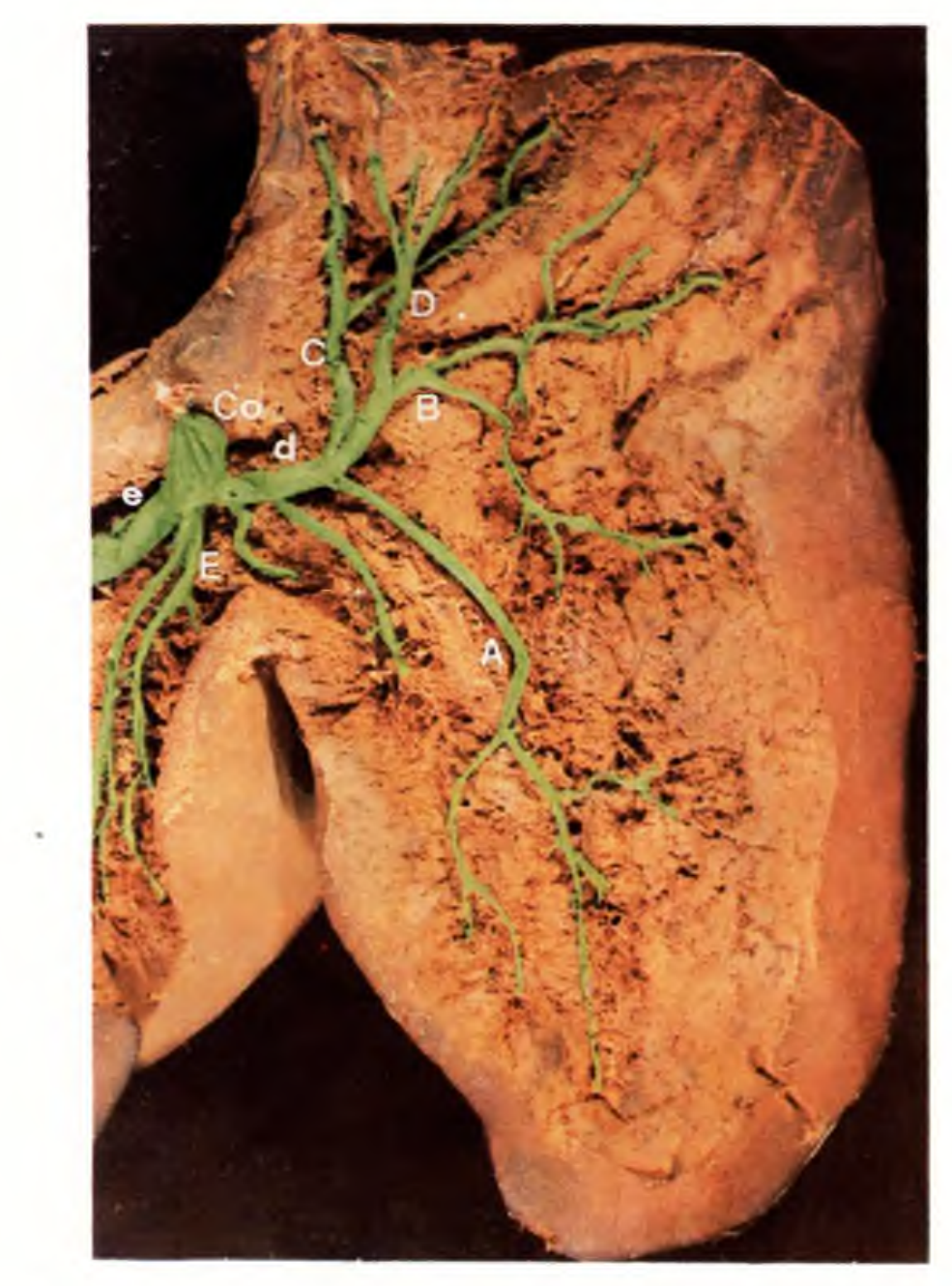

FIGURA 1 - Obs. $2 \mathrm{~m}$

Figado de eqüino Puro Sangue Inglês, macho, adulto, (partes direita e intermediária vistas pela fase visceral). 0 ductus choledocus ( $\mathrm{Co}$ ) resulta da tríplice convergência do ramus principalis dexter (d), ramus principalis sinister (e) e ramus lobi quadrati (E). O ramus principalis dexter (d) tem a constitui-lo o ramus medius lobi dextri (B) e o ramus processi caudati (D), integrando tronco para o qual convergem, por ordem, o ramus dorsalis lobi dextri (C) e o ramus ventralis lobi dextri (A). (redução de $4 \mathrm{x}$.)

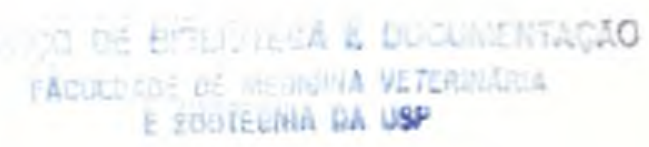




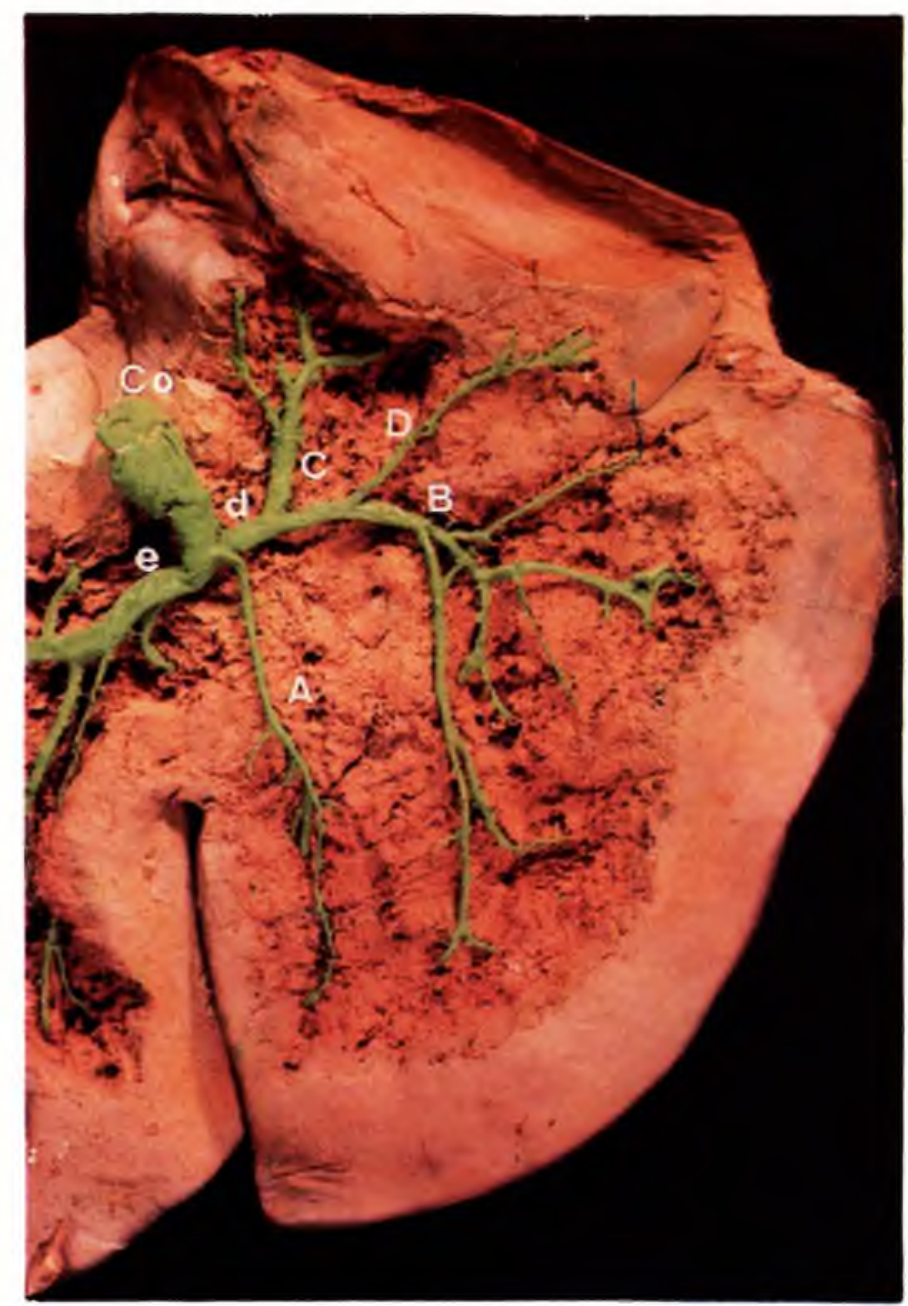

FIGURA 2 - Obs. 10m

Fígado de eqüino Puro Sangue Inglès, macho, adulto (partes direita e intermediária vistas pela face visceral). $\mathrm{O}$ ductus choledocus (Co) resulta da reunião do ramus principalis dexter (d), ramus principalis sinister (e) e ramus ventralis lobi dextri (A). O ramus principalis dexter (d) resulta da associação do ramus medius lobi dextri (B) e ramus processi caudati (D) compondo tronco no qual desembuca o ramus dorsalis lobi dextri (C). (redução de $4 \mathrm{x}$.) 


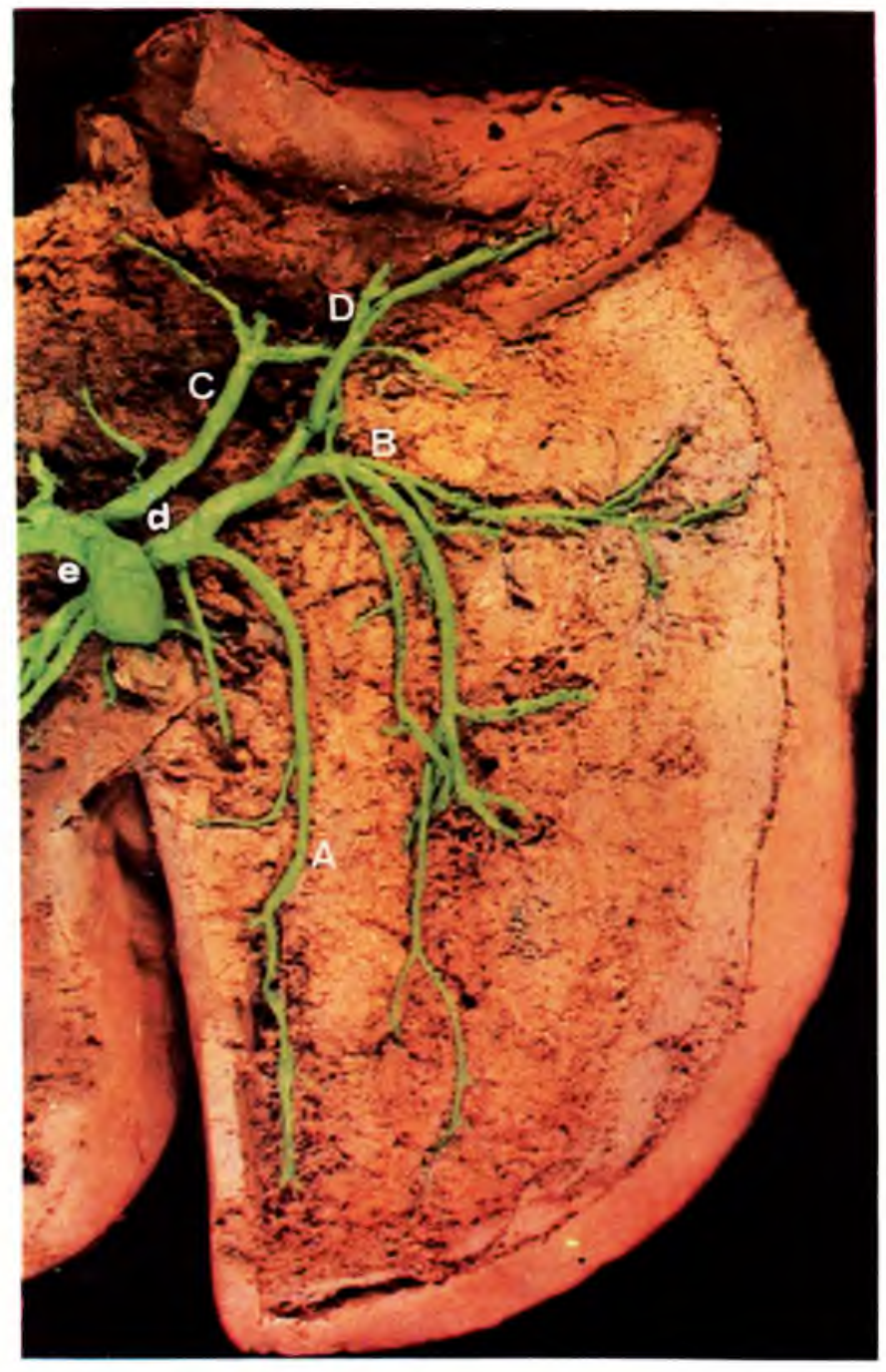

FIGURA 3 - Obs. $8 \mathrm{~m}$

Fígado de eqüino Puro Sangue Inglês, macho, adulto (partes direita e intermediária vistas pela face visceral). 0 ramus principalis dexter (d) resulta da confluência do ramus medius lobi dextri (B) e ramus processi caudati (D), constituindo tronco o qual acolhe o ramus ventralis lobi dextri (A). O ramus dorsalis lobi dextri (C) integra o sistema do ramus principalis sinister (e). (redução de $4 \mathrm{x}$.) 


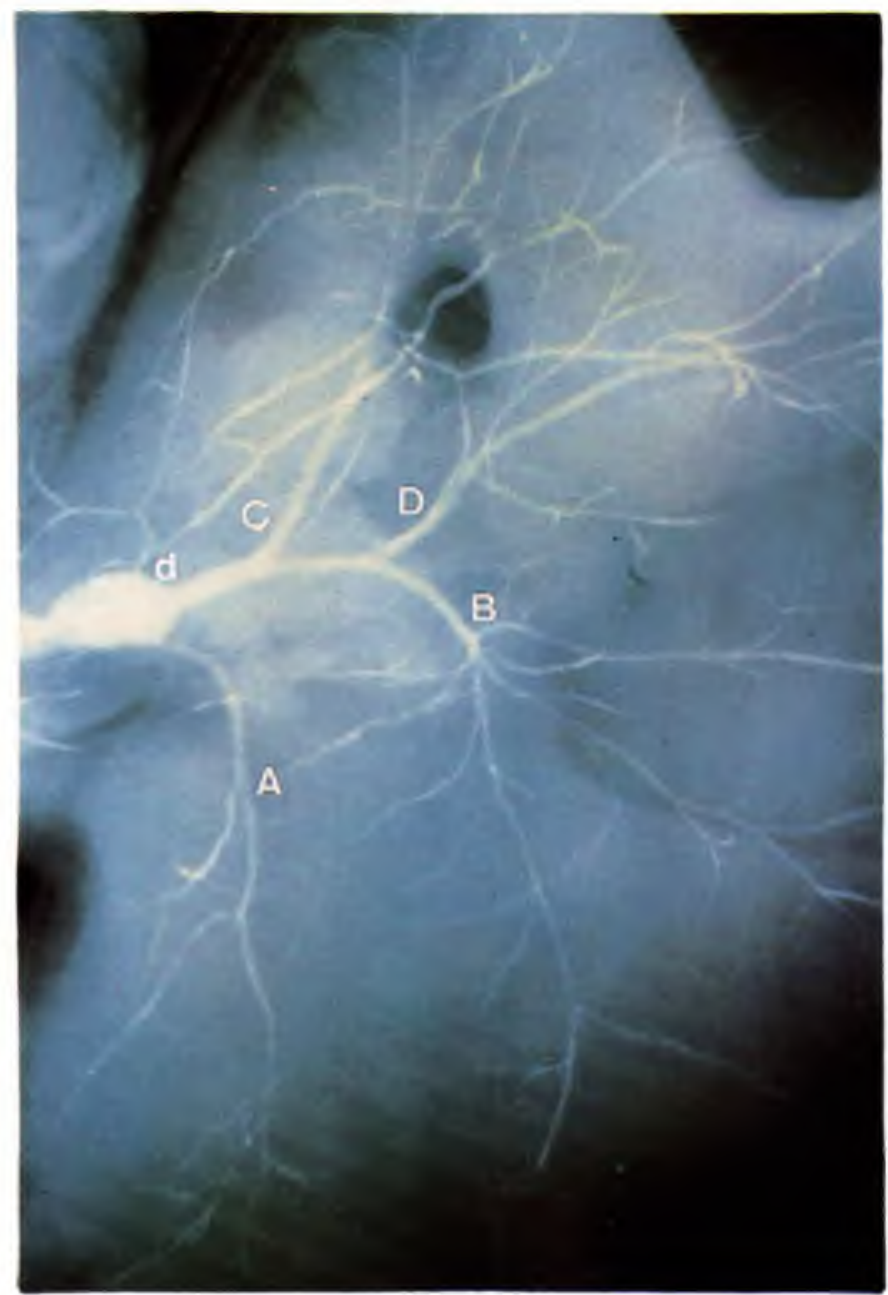

FIGURA 4 - Obs. $4 \mathrm{~m}$

Radiografia contrastada (Neoprene látex adicionado de Celobar) das vias biliferas de fígado de eqüino Puro Sangue Inglês, macho, adulto (parte direita vista pela face visceral). $\mathrm{O}$ ramus principalis dexter (d) resulta da união do ramus medius lobi dextri (B) e ramus processi caudati (D), originando tronco ao qual vem ter o ramus dorsalis lobi dextri (C) seguido do ramus ventralis lobi dextri (A). (redução de 3x.) 
1 - BOURDELLE, E \& BRE550U, c. Anatomie réglonale des animaux domestiques. Paris, J.B. Baillière, 1937, v.1, p. 51 .

2 - BRADLEY, D.C. The thorax and abdomen of the horse. Edinburgh, W. Green, 1922. p.123-124.

3 - BRUNI, R.C. \& ZIMMERL, U. Anatomia degll animali domesticl. 2.ed. Milano, Francesco Vallardi, 1947. $\checkmark .2,0.84$.

4 - CARADONNA, G.B. Apparechio gastropulmonare. In: ZIMMERL, U. Trattato di anatomia veterinaria. Milano, Francesco Vallardi, 1930, v. 2, p.563.

5 - DUPRAT, P. Segmentation hepatique chez le cheral. Alfort, 1970. 115p. These pour le doctorat - Ecole Nationale Vétèrinaire d'Alfort/.

6 - ellenberger, W. \& BAuM, H. Handbuch der vergleichenden Anatomie der Haustlere. 17. Auf. Berlim, Julius Springer, 1932. p.438-9.

7 - FAUILLI, N. L'anatomia del fegato nei principali animali domestici (cavallo, bue, malale, cane). Pisa, A. Cesari, 1923.

8 - FERREIRA, N. Contribuifzo ao estudo das vias billferas intra e extrahepaticas no Equus caballus. Sistematizacao do ramus principalis dexter; ramus principalis sinister e do ductus choledocus. Rev. Fac. Med. Vet. Zootec. Univ. 5. Paulo, $11: 201-26,1974$.

9- GETTY, R. Sisson and Grossman's the anatomy of the domestic animals. 5.ed. Philadelphia, W.B. Saunders, 1975. v.1, p.492-6.

10 - GONZRLEZ y GARCiA, J. \& GONZALEZ ALVAREZ, R. Anatomia comparada de los animales domesticos. 7.ed. Madrid, Gráficas Canales, 1961 p. 486 .
11 - JABLAn-PANTIC, 0. Caracteristics and comparative ratios of intrahepatic bile duct in domestic animals. Acta vet., Belgrada, 13:3-14, 1963.

12 - KOCH, T. Lehrbuch der VeterinarAnatomie. Jena, Gustav Fischer, 1963. v.2, p.127.

13 - LEPDUTRE, L. Notes du cours d'anatomie comparédes animaux domestiques. Gemblaux, J. Duculot, 1921. p. 147 .

14 - LEsBRE, F.X. Precis d'anatomie comparee des animaux domestiques. Paris, J.B. Baillière, 1922, v. 1 , p.658.

15 - MARTIN, P. Lehrbuch der Anatomie der Haustiere. Stuttgart, Schickhardt \& Ebner, 1915. v.2, p.50-1.

16 - montane, l. \& Bourdelle, e. Anatomie regionale des animaux domestlques. Paris, J.B. Bailliere, 1913, v.1, P.94.

17 - otTRUIANI, G. Richerche radiografiche comparative sulle vie bilifere intraepatiche. Atti Ist. Veneto Sci. Lett. Arti., 92:1085-7, 1933.

18 - SCHUMMER, A. \& NICKEL, R. Elngeweide. In: NICKEL, R.; SCHUMMER, A.; SEIFERLE, E. Lehrbuch der Anatomie der Haustiere. Berlim, Paul Parey, 1960. v.2, p.115-6.

19 - SCHWARZE, E. \& SCHRODER, L. Compendio de anatomia veterinária. Zaragoza, Acribia, 1970. v.2, p.104.

20 - SISSON, S. \& GROSSMAN, J.D. Anatomia de los animales domesticos. 4.ed. Barcelona, 5 alvat, 1969. p.419.

21 - ZANOLli, C. Manual de anatomia veterinaria. La Plata, Felix F. Santi, 1910. v. 1, p.345-6.

Recebido para publicacao em 11/12/1985 Aprovado para publicaça em 09/04/1986 Impresso em $12 / 86$ 